\title{
Überlegungen zum Verhältnis von Kapitalismus und Staatenkonkurrenz
}

\author{
Tobias ten Brink
}

Zusammenfassung: Das Spannungsfeld zwischen der sich globalisierenden Wirtschaftsentwicklung und dem Fortbestand des Staatensystems ist in vielen einseitig verallgemeinernden Thesen einer entstehenden Weltgesellschaft nicht hinreichend analysiert worden. Im Folgenden soll diesem Mangel mit einem politökonomischen Konzept begegnet werden. Um eine Untersuchung der vielfältigen Varianten internationaler Konkurrenzverhältnisse auch und gerade unterhalb der Schwelle der Gewaltanwendung zu ermöglichen, werden ferner ökonomische und geopolitische Konkurrenzbeziehungen sowie Spielarten der Geopolitik unterschieden.

Schlüsselwörter: Kapitalismus · Konkurrenz $\cdot$ Staatensystem · Außenwirtschaftspolitik · Geopolitik

\section{On the Relationship Between Capitalism and Inter-State Competition}

\begin{abstract}
The discrepancy between the globalising economic development and the continuity of the state system has so far not been adequately analysed. This is true, in particular, for the often one-sided works on an emerging world society. The following article seeks to counter this deficit with a concept based on theories of capitalism. To prepare the ground for an analysis of the various forms of competitive relations on the international level below the threshold of an open use of force, for instance in the field of foreign economic policy, economic and geopolitical forms of competition as well as varieties of geopolitics are differentiated.
\end{abstract}

Keywords: Capitalism · Competition $\cdot$ State system $\cdot$ Foreign economic policy $\cdot$ Geopolitics

\section{MPIfG Journal Article}

Tobias ten Brink: Überlegungen zum Verhältnis von Kapitalismus und Staatenkonkurrenz. In: Zeitschrift für Außen- und Sicherheitspolitik 5(1), 97 - 116 (2012). Springer VS

The original publication is available at the publisher's web site: http://dx.doi.org/10.1007/s12399-011-0243-2

The MPIfG Journal Articles series features articles by MPIfG researchers and visiting scholars published in peer-reviewed journals. Max Planck Institute for the Study of Societies (MPIfG) Cologne | www.mpifg.de

Online publiziert: 21.12 .2011

(C) VS Verlag für Sozialwissenschaften 2011

Dr. T. ten Brink $(\bowtie)$

Max-Planck-Institut für Gesellschaftsforschung,

Paulstraße 3, 50676 Köln, Deutschland

E-Mail: tenbrink@mpifg.de 


\section{Einleitung}

In dem vorliegenden Beitrag wird die These vertreten, dass weltwirtschaftliche Dynamiken und außenpolitische Strategien starker Staaten ein konfliktbeladenes Spannungsfeld bilden, das die Globalisierung als einen instabilen, fragmentierten Prozess erscheinen lässt. In vielen einseitig verallgemeinernden Thesen einer entstehenden Weltgesellschaft ist dies nicht hinreichend analysiert worden.

Innerhalb der Globalisierungsdebatte hatte in den letzten zwei Jahrzehnten eine bestimmte Harmonisierungsthese eine besondere Anziehungskraft entwickelt. Mit der zunehmenden Transnationalisierung der Weltwirtschaft sowie dem Wegfall des OstWest-Konfliktes ab 1989 sollte die „neue“ Weltordnung perspektivisch ein harmonisches, friedfertiges und demokratischeres Gesicht erhalten. Die Auffassung einer potentiellen „Pazifizierung“ der Weltgesellschaft prägte nicht nur volkswirtschaftliche Konvergenzvorstellungen der Neoklassik, sondern auch politikwissenschaftliche Theorien in der Disziplin der Internationalen Beziehungen (IB) oder avancierte Kosmopolitisierungsthesen (Beck 2004; Held und McGrew 2002; Ohmae 1995; Wendt 2003). Die optimistischen Erklärungen dieses „liberalen Postnationalismus“ (Müller 2009) gingen vielfach von einem langfristigen Versiegen der Quellen internationaler, mit staatlicher Gewalt verbundener Konflikte aus. Aktuelle Maßnahmen, wie offensive Währungspolitiken, Sanktionen, aber auch Militärinterventionen starker Staaten, werden vorwiegend als Antworten auf ,äußere“, den liberalen Kapitalismus bedrohende, archaische Verhaltensweisen bzw. als Ausdruck irrationaler Hegemonievorstellungen angesehen. Zahlreiche Autoren neigen dabei zur Überzeichnung einzelner Trends oder interpretieren diese zu einem nicht oder kaum aufzuhaltenden Übergang in eine „Zweite Moderne“, die die in Nationalstaaten zergliederte Welt ablöst (exemplarisch: Beck 2004). Im akademischen IB-Diskurs wurden diese Thesen bis vor Kurzem eigentlich nur von neorealistischen Argumentationen grundlegend bestritten. In dieser Perspektive werden die zuversichtlichen Aussichten einer Global Governance verneint und erhebliche Rivalitäten zwischen den Großmächten und zwischenstaatliche Konflikte bis hin zu Kriegen antizipiert (exemplarisch: Mearsheimer 2003).

In jüngerer Zeit - besonders unter dem Eindruck des „Kriegs gegen den Terrorismus", aber auch der Krisen der EU und der Welthandelsorganisation, den nationalen Aufstiegsprojekten in Indien oder China sowie der globalen Rezession 2008/2009 und den daran anschließenden nationalen Krisenlösungsstrategien - sind jenseits neorealistischer Positionen signifikante Modifizierungen in verschiedenen IB-Ansätzen vorgenommen worden (Aspekte der imperialen Ordnungspolitik und Unzulänglichkeiten in der Entstaatlichungsthese thematisieren beispielsweise: Brock 2006; Müller 2009; Rosenberg 2005. Vgl. ferner die in unterschiedlicher theoretischer Perspektive vorgetragenen Aufsätze zur fortwährenden Bedeutung des Staatensystems in: ten Brink 2011). Gleichwohl mangelt es in dieser neuen Diskurskonstellation an systematischen Auseinandersetzungen mit den Wechselverhältnissen zwischen dem globalen Kapitalismus und dem internationalen Staatensystem bzw. den hiermit verbundenen Konkurrenzeffekten. Dies mag teilweise durch eine disziplinäre Engführung erschwert werden, steht aber wohl auch mit einem noch nicht zureichend vollzogenen Wandel theoretischer Erwartungen in Verbindung, weshalb etwa die neue Weltwirtschaftskrise und die zumindest temporäre Aufwertung 
eines „starken“ Staates teilweise wie ein externer Schock behandelt werden und nicht bereits zu einer Modifikation der theoretischen Grundannahmen beigetragen haben.

Eine ähnliche Entwicklung hat sich in Ansätzen der Internationalen Politischen Ökonomie (IPÖ) vollzogen. Konstatierten diese vor dem Hintergrund der These einer „neoliberalen“ Wende ab den 1970er Jahren häufig eine dramatische Erosion von staatlichen Steuerungsfähigkeiten - Autorinnen und Autoren legten dem die Annahme der Entstehung einer transnationalen Managerklasse oder gar die Hypothese eines ,glatten“ Raums der Machtausübung, eines „Empire“, zugrunde (Cox und Schechter 2002; Hardt und Negri 2002; Strange 1996) - werden nun vermehrt die staatlich gesetzten Rahmenbedingungen des wirtschaftlichen Verkehrs sowie sicherheitspolitische Fragestellungen thematisiert (Möller 2010). Darüber hinaus kam es zur Wiederaufnahme und Weiterentwicklung politökonomischer und imperialismustheoretischer Ansätze, die jedoch im Mainstream der IB kaum Beachtung fanden (Callinicos 2009; Chibber 2004; Gindin und Panitch 2004; Harvey 2005; Hirsch 2005; Wood 2003).

An eine Reihe von Erkenntnissen in diesen (gleichwohl unterschiedlich konzeptualisierten) Perspektiven einer kritischen IPÖ wird im Folgenden angeknüpft. In einer integralen Perspektive verbindet der Autor dieses Beitrags zusätzlich weitere Forschungsergebnisse interdisziplinär miteinander - zum Beispiel politökonomische Überlegungen mit Ansätzen der materialistischen Staatstheorie sowie mit neogramscianischen, neopoulantzianischen und historisch-soziologischen Einsichten. Um einen strukturalistischen Bias zu vermeiden, wird eine historisierende Perspektive eingenommen. Im Gegensatz zu sozialwissenschaftlichen Analysen, in denen (wie in vielen Globalisierungsthesen) historische Entwicklungstrends entweder voreilig extrapoliert oder aber (wie in funktionalistischen Ansätzen) in den engen Bezugsrahmen einer kapitalistischen Entwicklungslogik eingeordnet werden, gilt es, internationale Konkurrenzmechanismen in ihrem historischen Wandel zu analysieren. ${ }^{1}$

Die im Folgenden entwickelten Überlegungen sollen eine Orientierung auf Konkurrenzverhältnisse und Konflikte ermöglichen, ohne in einen Reduktionismus macht- bzw. staatszentrierter Argumentationen zu verfallen. Die Fragmentierung des Staatensystems wird hierfür mit Merkmalen des globalen Kapitalismus in Zusammenhang gebracht (siehe Kap. 2). Um eine empirische Untersuchung der vielfältigen Varianten internationaler Konkurrenzverhältnisse auch und gerade unterhalb der Schwelle der offenen Gewaltanwendung zu ermöglichen, werden des Weiteren historisch variierende ,ökonomische“ und „geopolitische“ Konkurrenzbeziehungen sowie Spielarten der Geopolitik - verstanden als „harte“ bzw. „weiche“ Handlungsstrategien moderner Staaten zur Verteidigung, Befestigung bzw. Steigerung von Macht und Einfluss sowie zur nicht notwendigerweise direkten Kontrolle von Räumen - unterschieden (siehe Kap. 3 und 4). Im abschließenden Teil werden einige relevante außenwirtschaftspolitische Konfliktdynamiken der Gegenwart umrissen (siehe Kap. 5).

1 Da einige Argumente aus Platzgründen nur kursorisch eingeführt werden können, gebe ich Hinweise auf weiterführende Quellen. Vgl. für eine umfassendere Darstellung: ten Brink 2008b; ten Brink 2008a, S. 242-281. 


\section{Merkmale des globalen Geflechts vieler Kapitalismen}

Da der hier in groben Zügen eingeführte Ansatz quer zu anderen Zugängen steht, sind einige allgemein-theoretische Überlegungen unerlässlich. ${ }^{2}$ Wie Streeck (2009, S. 30) feststellt, können sozialwissenschaftliche Analysen insgesamt von einer stärkeren Berücksichtigung der historischen Besonderheiten der kapitalistischen Sozialordnung profitieren. Um die Eigentümlichkeit des modernen Staatensystems herauszuarbeiten, werden daher im Folgenden Zusammenhänge mit der dominierenden gesellschaftlichen Form unserer Zeit, dem globalen Kapitalismus und den mit ihm verbundenen Konkurrenzverhältnissen und Hierarchien, herausgestellt.

Dabei besitzt die Weltwirtschaft eine konstitutive Bedeutung für das Verständnis der fragmentierten Weltordnung. Der real existierenden Weltwirtschaft fehlt ein Steuerungszentrum. Der Weltmarkt entsteht aus dem Zusammenwirken einer unüberschaubaren Anzahl von Faktoren, die sich von den beteiligten Akteuren nicht angemessen durchschauen lassen. Er besteht als global wirksamer Handlungszusammenhang und übt erhebliche Handlungszwänge aus. Die Reproduktion der Weltwirtschaft in ihrer Gesamtheit verkörpert insofern einen Prozess ohne steuerndes Subjekt, der unzählige nicht-antizipierte, paradoxe Effekte zeitigt.

Die sich steigernde wechselseitige Abhängigkeit sozio-ökonomischer Prozesse, die in der Globalisierungsdebatte mitunter als das Ergebnis von kaum bestimmten Modernisierungskräften beschrieben wurde, bildet nur eine Seite des kapitalistischen Reproduktionsprozesses. Seine eigentümliche Form entwickelt er erst im Rahmen der wettbewerbsgetriebenen Akkumulation, der Konkurrenz zwischen den Produktionseinheiten, die eine ungleichzeitige, kombinierte und krisenhafte Dynamik befördert:

Als Gesamtresultat [...] strebt der Kapitalismus in seinem ständigen Durst nach unendlicher Kapitalakkumulation stets die Errichtung einer geographischen Landschaft an, die seine Aktivitäten zu einem gegebenen Zeitpunkt erleichtert, nur um sie zu einem späteren zerstören und eine ganz andere Landschaft aufbauen zu müssen. Solcherart ist die Geschichte der schöpferischen Zerstörung eingeschrieben in die Landschaft der tatsächlichen historischen Geographie der Kapitalakkumulation. (Harvey 2005, S. 102)

Eine Folge dieses anarchischen Prozesses ist die Herausbildung neuer Wachstumsräume, die sich zu wirtschaftlichen und politischen Machtpolen entwickeln können und in dieser Weise gefestigte internationale Kräftekonstellationen in Frage stellen (van der Pijl 2006). Gegenwärtig begünstigt eine Restrukturierung des produktiven Kapitals in Richtung Ostasien besonders Chinas nachholende kapitalistische Entwicklung - der asiatische Anteil am weltweiten Bruttosozialprodukt ist zwischen 1960 und heute von $13 \%$ auf etwa $30 \%$ gestiegen - und verleiht dem bevölkerungsreichsten Land der Erde den Status eines überaus relevanten strategischen Standorts der einstigen Peripherie.

Um die mit der Entwicklungsdynamik des globalen Kapitalismus verbundene Instabilität genauer zu fassen, werden nun zunächst historisch variable Merkmale einzelner kapi-

2 Viele der nachfolgenden Argumente bewegen sich auf einem hohen Abstraktionsniveau; empirisch illustriert werden sie nur an einigen Stellen. 
talistischer Systeme ${ }^{3}$ beschrieben: Konkurrenzverhältnisse zwischen Unternehmen und Krisendynamiken (siehe Kap. 2.1), die ,vertikale“ Konfliktachse der Arbeitsbeziehun-gen (siehe Kap. 2.2), die Interaktion zwischen ökonomischen und politischen Akteuren sowie die Einbettung einzelner Volkswirtschaften in weltwirtschaftliche und -politische Zusammenhänge (siehe Kap. 2.3) sowie Geldverhältnisse bzw. Finanzsysteme (siehe Kap. 2.4). ${ }^{4}$

Die verschiedenen Merkmale des Kapitalismus - oder genauer, der „,vielen“, real existierenden Kapitalismen - befördern nicht nur internationale wirtschaftliche Abhängigkeiten und Konkurrenzverhältnisse, sondern auch staatliche (Standort-)Strategien und (geo-)politische Konflikte.

2.1 Die horizontale Achse kapitalistischer Konkurrenzbeziehungen und die Rolle der krisenhaft-ungleichen (welt-)wirtschaftlichen Entwicklung

Die systemische Notwendigkeit der erweiterten Akkumulation und Innovation im Kapitalismus setzt sich vermittelt durch die ,horizontale“ Konkurrenz der Unternehmen durch. Diese wirkt als sozialer Sanktionsmechanismus, der Unternehmen dem Zwang zur Akkumulation bei Strafe der Existenzgefährdung unterwirft. Kapitalistische Märkte entstehen in der Regel in einer spontanen, dezentralen Weise und sind nur bedingt steuerbar (Mann 1990, S. 24). Die kapitalistische Interdependenz wird daher nicht automatisch Garant der sozialen Integration, sondern birgt erhebliche Instabilitäts-, Fragmentierungs- und Krisenrisiken in sich. Die maß- und endlose Kapitalanhäufung sowie der Mechanismus der Konkurrenz verweisen auf die Grenzen der Steuerung, auch und gerade auf globaler Ebene.

In neoklassischen Modellen wird der Wettbewerb als eine Kraft angesehen, die die Beziehungen zwischen den Kapitaleinheiten harmonisiert und Unterschiede zwischen Unternehmen, Regionen und letztlich auch Staaten nivelliert. Diese Konvergenzthese beruht allerdings auf unhaltbaren Prämissen - wie etwa der Annahme der vollständigen Konkurrenz und Markttransparenz, homogener Güter, konstanter Produktionstechnik sowie Nachfragestruktur. Die Neoklassik ignoriert die raumzeitliche Bewegung der Unternehmen, ihre variierende institutionelle Einbettung sowie die Realität von ungleich entwickelten Kapitalien aufgrund ungleich entwickelter Produktivitätsniveaus. Die Expansion des Kapitalismus bringt aus diesem Grund gravierende raumzeitliche Ungleichheiten nicht nur zwischen der „OECD-Welt“" und Schwellen- bzw. Entwicklungsländern, sondern auch innerhalb ersterer hervor. Akkumulationsprozesse setzen sich ungleichmäßig durch, weil die verschiedenen nationalen Gesellschaften unterschiedliche Voraussetzungen für die Etablierung und Reproduktion kapitalistischer Produktionsverhältnisse bieten (Block 2005).

Eine Analyse der kapitalistischen Produktions-, Konsumtions- und Verteilungsprozesse sowie ihrer institutionellen Einbettung legt zugleich grundlegende Widersprüche des Wirtschaftskreislaufs offen. Historisch traten diese als Überakkumulations-, Unter-

3 Davon zu unterscheiden ist der Begriff der kapitalistischen „Gesellschaft“. Letztere umfasst weitere Dimensionen (z. B. Hausarbeit, Familie, soziokulturelle Phänomene).

4 Die Unterscheidung historisch variabler Dimensionen des Kapitalismus geht $u$. a. auf regulationstheoretische Überlegungen zurück (vgl. Boyer 2005; ten Brink 2008a, S. 60-135). 
konsumptions-, Disproportionalitätskrisen sowie Krisen des Kredit- und Finanzbereichs auf (Harvey 2010). Die Tatsache des relativ simultanen und allgemeinen Charakters besonders der „großen“ Krisen des Kapitalismus in den 1870er, 1930er und 1970er Jahren (und allem Anschein nach auch gegenwärtig) - obgleich sie auf verschiedenartige sozioökonomische, politische und kulturelle Bedingungen in unterschiedlichen Gesellschaften trafen - verweist darauf, dass nationale Akkumulationsregime auf vielfältige Weise mit der internationalen Konkurrenz (und inter- wie transnationalen Politiken) verbunden sind (Brenner 2006).

Wie der weitere Argumentationsgang nahelegt, resultieren aus der Dynamik der Kapitalakkumulation eine Reihe von außen(wirtschafts-)politisch vermittelten Wettbewerbs- und Krisenlösungsstrategien. Relevant für die vorliegende Fragestellung sind beispielsweise die internationale Verlagerung von Kapital, die kapitalistische Landnahme in der Form der Erschließung neuer Märkte, die Sicherung von Rohstoffquellen, Pipelinetrassen etc. sowie die Lenkung von Investitionen.

\subsection{Die vertikale Achse kapitalistischer Sozialbeziehungen}

Die Konflikthaftigkeit kapitalistischer Systeme erschöpft sich nicht in den horizontalen Konkurrenzbeziehungen zwischen Unternehmen. Diese werden von der „,vertikalen“ Konfliktachse der Arbeitsbeziehungen und Eigentumsverhältnisse überlagert. Soziale Stratifizierungen und insbesondere die vertikale „Klassenpolarisierung zwischen Vermögensbesitzern und Vermögenslosen und die durch sie geschaffene sozialstrukturelle Spannung“ stellen erst einmal eine „objektive Grundbedingung kapitalistischer Dynamik“ dar (Deutschmann 2009, S. 38). Deutschmann zufolge gäbe es ohne diese Spannung kein Aufstiegsmotiv und auch keine Innovationskonkurrenz. Moderne Wirtschaften beruhen auf der privaten Aneignung der produzierten Waren, die nicht auf einem zuvor hergestellten gesellschaftlichen Konsens gründet. Mit den hierarchisch strukturierten Arbeitsverhältnissen sind spezifische Eigentums- und Verfügungsrechte verbunden, die die Partizipation der sozialen Akteure an den Entscheidungs-, Planungs- und Kontrollprozessen über Produktion, Verteilung und Konsumtion maßgeblich bestimmen. Die vertikale Achse kapitalistischer Sozialbeziehungen bildet aus diesem Grund nicht nur in Krisen eine Basis fortwährender innergesellschaftlicher Auseinandersetzungen.

Um soziale Konflikte einzudämmen, sind interne gesellschaftliche Krisen und Legitimationsdefizite geschichtlich vielfach von Machteliten dadurch zu lösen versucht worden, dass ein externes „Anderes“ konstruiert wurde, um eine Krisenexternalisierung zu bewirken. Ein innenpolitischer Konsens über Klassengrenzen hinweg wird über eine Abgrenzung nach außen herzustellen versucht. Wie neogramscianische und neopoulantzianische Arbeiten verdeutlichen, lässt sich die Außenpolitik einer Staatsführung mit anderen Worten nicht losgelöst von einer Analyse der Dynamiken in nationalen Wirtschaften verstehen, u. a. von hegemonialen Projekten sowie von sozioökonomischen Desintegrationstendenzen und hiermit in Verbindung stehenden nationalistischen Bewegungen (Brand 2006; Cox 1987; De Graaff und van Apeldoorn 2011). Die Bedeutung kollektiver Identitäten, affektiver Dimensionen des Politischen sowie von Exklusion als Ausdrucksformen antagonistischer Verhältnisse darf auch in liberalen Demokratien nicht zu gering geschätzt werden (Geis 2008; Mouffe 2007, S. 64-67). 


\subsection{Staat und Staatensystem: Die Interaktion wirtschaftlicher und politischer Akteure}

In welcher Weise ist die ökonomische Konkurrenz, Krisenhaftigkeit und grenzenübergreifende Suche nach rentablen Investitionen mit der Politik von Staaten verbunden? Zur Beantwortung dieser Frage müssen die Interdependenzen zwischen Politik und Wirtschaft sowie die Ursachen für die Existenz vieler Einzelstaaten untersucht werden.

Kapitalistische Wirtschaftssysteme sind zu ihrer Reproduktion auf „nichtökonomische“ Institutionen angewiesen. Dazu gehört insbesondere der moderne Staat. Historisch betrachtet konnte eine gelingende Kapitalakkumulation ohne eine relativ autonome politische Instanz, die das Gewaltmonopol innehat, über einen längeren Zeitraum nicht realisiert werden. Der Staat stellt zwar keine homogene Einheit dar, versucht jedoch gleichwohl eine Reihe von sozialen, rechtlichen und infrastrukturellen Integrations- und Anpassungsleistungen zu garantieren, die die Aufrechterhaltung kapitalistischer Vergesellschaftung erst ermöglichen (z. B. Vertragsverhältnisse, Infrastrukturmaßnahmen, Ausbildungssektor). Wie in der materialistischen Staatstheorie veranschaulicht wird, bilden Wirtschaft und Politik derart ein Regulierungsgeflecht, welches durch strukturelle Interdependenzen gekennzeichnet ist: Staatliches Handeln spielt bei der Gestaltung wirtschaftlicher Vorgänge eine konstitutive Rolle. Doch nicht nur die Unternehmen sind abhängig von den jeweiligen staatlichen Instanzen, umgekehrt hängt die Existenz des Einzelstaates von den erfolgreichen Aktivitäten der jeweiligen nationalen Wirtschaft ab (Block 1994, S. 696-705; Jessop 2007; Offe und Ronge 1976). ${ }^{5}$

Welthistorisch hat sich auf diese Weise ein sich unterscheidendes, aber miteinander verbundenes und durch ständige Differenzierungs- und Anpassungsprozesse gekennzeichnetes Geflecht „politischer“ Ökonomien herausgebildet, die sich in einem „Standortwettbewerb“ gegenüberstehen. ${ }^{6}$ Die kapitalistische Entwicklung mündete nicht in die Organisation eines Weltstaates ein. Es koexistieren viele Staaten, was auf wenigstens zwei zentralen Ursachen beruht: Erstens erfordert die Notwendigkeit der Schaffung klassenübergreifender Koalitionen zur Herstellung innergesellschaftlicher Stabilität die Integrationsleistungen der Einzelstaaten (siehe Kap. 2.2). Ohne die Existenz vieler Einzelstaaten als rechtlich verfasste und symbolische Referenzsysteme wären grundlegende Mechanismen der Ausbalancierung von innergesellschaftlichen Konflikten kaum zu gewährleisten. Diese Form der kollektiven Identitätsbildung setzt andere Staaten gewissermaßen voraus. Die Beseitigung ,nationaler“ Spaltungen würde etwa die Wirkmacht wesentlicher sozia-

5 Im Folgenden wird von der sozialen Konstruktion der Grenzziehungen zwischen öffentlichen und privaten Sektoren abgesehen und die gegenwärtige Rolle staatlicher Akteure dargestellt.

6 Die territoriale Fragmentierung ging der Entstehung des Kapitalismus historisch voraus. Inwieweit das fortwährende Bestehen des Staatensystems ein gewissermaßen kontingentes Residuum darstellt oder aber in das kapitalistische Weltsystem als Konstituens integriert wurde, ist Gegenstand von Auseinandersetzungen: vgl. für erstere Position: Teschke 2003; Wood 2003; für letztere Position: Callinicos 2007; Hirsch 2005. Der hier vorgestellte Ansatz repräsentiert eine „Zwischenposition“ insofern, als ich eine Auflösung des Staatensystems für höchst unwahrscheinlich halte, hierfür jedoch „historische“ und nicht in erster Linie „strukturelle“ Argumente heranziehe. 
ler Integrationsmodi außer Kraft setzen (Anderson 1996; Görg 2002). ${ }^{7}$ Zweitens hat die wirtschaftliche Entwicklung nicht zu einer Homogenisierung auf globaler Ebene geführt. Die Welt ist kein glatter, sondern ein gekerbter Raum. Es haben sich fest verankerte wirtschaftliche Zentren gebildet, die in einem engen Wechselverhältnis zu den auf dem jeweiligen Territorium etablierten Staatsapparaten stehen. Relativ immobile raum-zeitliche Fixierungen des Kapitals, insbesondere des territorial verankerten Produktivkapitals, stellen hohe Anforderungen an die staatlichen Regulierungsapparate (Callinicos 2009, S. 73-100; Harvey 2005, S. 95-102; Mann 1997). ${ }^{8}$

Gleichwohl diese Konstellation eine historische gewordene ist, wie funktionalistischen Annahmen entgegnet werden muss, kennzeichnet diese dennoch die Gegenwart wie kaum ein anderer Sachverhalt. Ein Ende des Staatensystems ist theoretisch vorstellbar, doch praktisch nicht abzusehen: Ist der Kapitalismus einmal historisch in nationalen Formen entstanden, so Wood (2003, S. 137-142, S. 154-159), und wurde er in anderen national organisierten Prozessen imitiert, reproduziert sich dieses System mit einer hohen Wahrscheinlichkeit (vgl. Lacher 2005). Zudem sorgt das Unvermögen supra- bzw. transnationaler Organisationen, die Widersprüche des globalen Kapitalismus erfolgreich zu regulieren, für den Beibehalt des zwischenstaatlichen Systems (Brand 2006). ${ }^{9}$

Um eine gewisse Sicherheit zu gewährleisten, müssen wirtschaftliche Verflechtungsräume ein Maß an Berechenbarkeit aufweisen, die von staatlichen Garantien und Hilfestellungen abhängt. Komplexe Produktionsstrukturen erfordern eine administrativordnungssetzende Infrastruktur (wie Rechtswesen, Verwaltung, Lizenzierungsverfahren), eine wirtschaftliche Infrastruktur (etwa Transportwesen, Kommunikation) sowie weitere Regulierungen (beispielsweise im Arbeitsrecht oder bei der Regelsetzung für ökonomische Transaktionen). Ferner verlangen die Innovationen der Produktionsprozesse eine

7 Vgl. zur Kritik des Konzepts der Denationalisierung und zur Beschreibung von Entwicklungstendenzen in Situationen, in denen Staat und Nation auseinanderfallen, Müller (2009, S. 225228).

8 Die quantitative Bedeutung der fixierten Produktionsmittel übertrifft das BIP von Volkswirtschaften. Das Bruttoanlagevermögen in Deutschland, ein grober Indikator, lag laut Statistischem Bundesamt 2009 bei 10,7 Bio. $€$ im Vergleich zu einem jährlichen BIP von etwa 2,5 Bio. $€$.

9 In neopoulantzianischen Ansätzen wird die Rolle von internationalen politischen Institutionen unter Bezugnahme auf Klassenstrategien und gesellschaftliche Kräfteverhältnisse analysiert (vgl. Hirsch 2005). Internationale Institutionen bilden demnach Verdichtungen konfligierender und nur teilweise in einen Kompromiss einzubindender Interessen ab. Da diese Interessen auf der einzelstaatlichen Verdichtung von Kräfteverhältnissen aufbauen, handelt es sich um die „Verdichtung von Kräfteverhältnissen zweiter Ordnung“ (Brand 2006, S. 267). Internationale politische Institutionen mögen mehr und mehr den nationalen Regierungen vergleichbare Aufgaben übernehmen - ohne eine substantielle Ausweitung sowohl ihrer materiellen Ressourcenbasis als auch ihrer demokratischen Legitimität werden sie nur schwerlich die Aufgabe der Herstellung globaler gesellschaftlicher Integration leisten können. Zwar lassen sich Tendenzen zur Transnationalisierung von Machteliten (van der Pij1 2007) nachweisen, doch handelt es sich eher um fragile Formen von kooperativem inter- und/oder transnationalem sozialen Handeln. Diese intergesellschaftlichen Beziehungen sind in vergleichsweise geringem Maße institutionell gefestigt. Die neuartigen Formen der Global Governance können derart als institutionalisierte Politikkoordinierung, in der aber die Konkurrenz nicht aufgehoben, sondern fortgesetzt wird, analysiert werden. 
gewisse Risikoübernahme durch den Staat mitunter mittels Subventionen oder Direktbeteiligungen. Zugleich sind die Staaten angehalten, für ein Mindestmaß an ausgebildeten Arbeitskräften und deren soziale Sicherheit zu sorgen. Dass die Wachstumsstrategien der Unternehmen einer erheblichen Hilfestellung durch politische Regulierung bedürfen, lässt sich u. a. an einer kontinuierlich hohen Staatsquote, auch in sogenannten „,neoliberalen" Volkswirtschaften, ablesen (Hay 2005).

Umgekehrt sind die Staatsapparate abhängig von einer gelingenden Akkumulation innerhalb ihres Hoheitsgebietes, was sich u. a. in dem Interesse am Erhalt der Besteuerungsgrundlagen ausdrückt: Um handlungsfähig zu bleiben, müssen die politischen Eliten der Tatsache Rechnung tragen, dass die fiskalischen Ressourcen und damit die Mittel, staatliche Politik gestalten zu können, letztlich von einer einigermaßen reibungslosen wirtschaftlichen Entwicklung abhängen. Auch wenn Firmen mehr und mehr global agieren, gehen die Staatsführungen daher von international wettbewerbsfähigen ,nationalen“ bzw. „einheimischen“ Unternehmen aus und haben ein Interesse an dauerhaften Beziehungen zu ihnen. ${ }^{10}$ Aus diesem Grund verfolgt eine Regierung dieses Ziel in der Regel nicht nur, weil ihre Mitglieder durch Lobbying bestochen oder mit Wirtschaftseliten personell verflochten sind. ${ }^{11}$

Die Globalisierung bringt also eine Gegentendenz hervor: Die transnationale Akkumulation ist im Zuge permanenter Ortswechsel und der Suche nach profitablen Anlagemöglichkeiten gebunden an eine auch staatlich produzierte geographische Infrastruktur. Die Tendenz der Unternehmen, sich weltweit auszubreiten, setzt eine lokale Fixierung von Kapitalinvestitionen voraus. Die De-Territorialisierung geht einher mit einer Produktion territorialer Fixierungen, die zu unterstützen der Einzelstaat besonders geeignet ist (Jessop 2007, S. 180-197). Der globale Kapitalismus ist demzufolge bis dato nur als eine Vielzahl politischer Ökonomien angemessen zu konzeptualisieren.

Inwiefern sich der Prozess der Reartikulation des Raumes sowie seiner Regulierung durch ,viele“ Kollektivakteure in politische „Staatsprojekte“ transformiert und diese wiederum in konträre Positionen zu anderen Staatsprojekten geraten, kann freilich erst unter Berücksichtigung der Ausprägungen in spezifischen historischen Phasen und konkreten historischen Konstellationen näher untersucht werden.

10 Es ist aus diesem Grund voreilig, von den hohen Transnationalisierungsgraden der größten Konzerne auf die Struktur einzelner Volkswirtschaften zu schließen. Es muss zudem das Verhältnis mikroökonomischen Handelns und makroökonomischer Zusammenhänge berücksichtigt werden. Eine Konzentration auf die konkreten Träger mikroökonomischer Prozesse (Unternehmen) und deren globale Ambitionen darf nicht zur Ausblendung der fragmentierten makroökonomischen Markt- und Verwertungsgefüge sowie deren politischer Einbettung in viele separate Herrschaftsräume führen. Die Aggregierung mikroökonomischen Verhaltens lässt keine einfachen Rückschlüsse auf makroökonomische Zusammenhänge zu. Zwischen den Interessen von transnational orientierten Unternehmen (bzw. Managergruppen) und dem wirtschaftspolitischen, stärker von makroökonomischen Erwägungen geleiteten Handeln staatlicher Machteliten können erhebliche Differenzen bestehen.

11 Parallel hierzu bilden Einzelstaaten in einer nicht unmittelbar ökonomisch ableitbaren, sondern auch auf ihre Selbsterhaltung bezogenen Weise grundlegende Interessen an der Attraktivität der von ihnen gesicherten und angebotenen „Standortvorteile“ aus. 


\subsection{Eine Welt, viele Währungen: Kapitalistische Geld- und Finanzsysteme}

Ein weiteres Merkmal des Kapitalismus, das internationalen Austausch ermöglicht, ihn jedoch zugleich konflikthaft strukturiert, sind die Geld- und Währungsverhältnisse. Das Geld ist ein grundlegendes Medium der Vergesellschaftung in der kapitalistischen Wirtschaft. Der Kreislauf des industriellen Kapitals gewinnt durch den Mechanismus des Kredits bzw. des Verkaufs von Aktien seine Elastizität. Zugleich unterliegen kapitalistische Akteure sowie die Tätigkeit des Staates, der sich seine materiellen Ressourcen in Geldform beschafft, einer Geldbeschränkung.

Da das Geld immer bezogen ist auf konkrete einzelstaatliche (im Falle des Euros, überregionale) Währungsräume und die Geldpolitik mit dem Gültigkeitsbereich einer Währung Binnen- und Außenwirtschaft gegeneinander abgrenzt, erhält das Geld den Status eines politisch regulierten Mediums, was besonders für die Geldschöpfung zutrifft (Cohen 2000, S. 131-149; Mosley 2003). ${ }^{12}$ Innerhalb einzelner Währungszonen wirken Zentralbanken als Emissionsstelle und als ,lender of last resort“. Zudem spielen sie eine Rolle bei der Regulation des internationalen Zahlungsverkehrs. ${ }^{13}$ Mangels eines Weltstaates sind es jedoch ,zwingend nationale Währungen, die internationale Funktionen übernehmen müssen, was eine Reihe von Problemen aufwirft" (Herr und Hübner 2005, S. 99).

Die Stabilität des jeweils eigenen Währungsraums bildet eine wichtige Komponente des globalen Wettbewerbs. Da die verantwortlichen Institutionen diese im Verhältnis zu den jeweiligen anderen Währungen durchsetzen müssen - etwa durch Strategien der „Unterbewertung“ - entsteht eine Währungskonkurrenz, die bis zum geopolitischen Währungskonflikt eskalieren kann.

\section{Internationale Konkurrenzverhältnisse}

Im Folgenden werden zwei zentrale Formen des Wettbewerbs auf globaler Ebene beschrieben, die aus dem Geflecht ,vieler“ politischer Ökonomien historisch entstanden sind. Diese fasse ich als Überschneidung zweier, relativ unabhängig voneinander existierender und nicht aufeinander zu reduzierender, jedoch sich zeitweise verschränkender Muster der soziö̈konomischen Unternehmens- sowie der geopolitischen Staatenkonkurrenz auf. Weil diese Formen des Wettbewerbs sich unterscheiden und differierende Akteure in sie involviert sind, schwankt deren Interaktion historisch betrachtet. Unternehmen und Staatsführungen orientieren sich an verschiedenartigen Kriterien der Reproduktion und

12 Hegemoniale Leitwährungskonstellationen (Goldstandard und das englische Pfund als Leitwährung vor 1914, Bretton-Woods-System und US-Dollar als Leitwährung des Westens nach 1945) sind vor dem Hintergrund internationaler Machtverschiebungen von Oliogopolkonstellationen (etwa die Zeit zwischen 1914 und 1945) abgelöst worden.

13 Über die genannten Handlungsmöglichkeiten verfügen nicht alle Einzelstaaten gleichermaßen souverän. Währungen von starken Staaten übernehmen Geldfunktionen in schwächeren Staaten. Zugleich sind die Staaten nicht die alleinigen Akteure in diesem ,strategischen Spiel““. Die Entscheidung über die Stellung einer nationalen Währung in der Hierarchie der Währungen wird heute auch von privaten Akteuren auf den Devisenmärkten getroffen. 
bilden somit untereinander ein Spannungsverhältnis aus, das regelmäßig divergierende Handlungsstrategien zur Folge hat (vgl. ten Brink 2008b, S. 118-130). ${ }^{14}$ Das wesentliche ökonomische Kriterium der Reproduktion besteht in der Behauptung der relativen Kapitalstärke und damit der Profitabilität; sollten Einzelkapitalien dieses Ziel verfehlen, drohen der Bankrott oder die Übernahme. Das wesentliche Kriterium der politischen Reproduktion zielt dagegen zunächst einmal darauf, die Herrschaft gegenüber der jeweiligen Bevölkerung und gegenüber anderen Staaten sowie äußeren sozialen Kräften zu behaupten. Hier droht im Falle des Scheiterns eine Delegitimierung der Herrschaft.

Die wechselseitige strukturelle Abhängigkeit beider Akteursebenen führt aber immer wieder auch zu kongruenten Handlungsstrategien, die sich unter anderem in der politischen Hilfestellung bei der globalen Restrukturierung der Kapitalverwertung und dem Versuch des Managements der internationalen Beziehungen ausdrücken. Als Beispiele hierfür gelten die wirtschaftspolitische Unterstützung bei der Durchführung von Auslandsinvestitionen oder diplomatisch vermittelte Wirtschaftsbeziehungen. Ebenso spielen die militärischen Kapazitäten und Sicherheitsstrategien von Staaten als „diskrete“ Hintergrundinformation über ihr internationales Durchsetzungsvermögen eine Rolle, was wiederum Vorteile für wirtschaftliche Akteure mit sich bringen kann - etwa den Zugang zu vormals geschlossenen Märkten.

Sollten die Akkumulationsprozesse von Unternehmen die Grenzen der Volkswirtschaft überschreiten, ist die Frage, wie sich dies auf die Handlungsstrategien des ursprünglichen Standorts auswirkt. Historisch wurden internationale Austauschverhältnisse häufig durch die Schaffung suprastaatlicher Abkommen bzw. internationaler politischer Institutionen (temporär oder längerfristig) reguliert. Ist es für den Staat jedoch absehbar, dass die Internationalisierungsprozesse seine Macht potentiell unterminieren könnten, ringt er mit einer gewissen Wahrscheinlichkeit darum, Investitionsströme mit viel „Mühe und Besonnenheit zu seinem eigenen Vorteil“" zu lenken, sowohl intern als auch extern:

Und was den externen Bereich angeht, wird er typischerweise große Aufmerksamkeit auf die Asymmetrien legen, die immer aus dem Handel zwischen Räumen

14 Teschke und Lacher haben in einer Auseinandersetzung mit Callinicos (2007), der eine ähnliche Unterscheidung trifft, darauf verwiesen, dass ein derartiger Ansatz droht, in einen machtpolitischen Realismus zurückzufallen: ,the theoretical ascription of one generic rationality of permanent politico-territorial (imperial) accumulation to state managers seems to us as being [...] fraught with dangers of reification (constituting also an unnecessary relapse into realist verities) [...]. This is not to deny that 'the logic of anarchy' and 'the logic of capital' exercise certain pressures, but the ways in which states or firms react to these pressures cannot simply be derived from these 'imperatives', but always depend on a variety of factors" (Teschke und Lacher 2007, S. 570; vgl. zur Kritik an Harveys Diskussion territorialer Machtstrategien: Jessop 2004, S. 12). Die historische Variabilität staatlicher Strategien, die kontingente Konstruktion territorialer Projekte etc. dürfen nicht vernachlässigt werden (vgl. ten Brink 2008b, S. 221-263).

Ferner muss konzediert werden, dass internationale Beziehungen erst dann hinreichend zu erfassen sind, wenn diese nicht nur klassisch als ökonomische und politische, sondern auch als „intergesellschaftliche“ Beziehungen verstanden werden (Rosenberg 2006). Das Intergesellschaftliche geht in der Weltwirtschaft und im Weltstaatensystem nicht auf. Es umfasst ebenfalls soziale, politische, soziokulturelle und normative Dimensionen jenseits des Staates und der Wirtschaft. 
entstehen, und versuchen, die Trümpfe der monopolistischen Kontrolle so stark zu machen, wie er kann. Er wird sich, kurz gesagt, notwendig am geopolitischen Kampf beteiligen und wo er kann auf imperialistische Praktiken zurückgreifen. (Harvey 2005, S. 108) $)^{15}$

Die geopolitischen Anstrengungen moderner Staaten sind somit nicht nur als Ausdruck der Sonderinteressen eines militärisch-industriellen Komplexes oder irrationaler Machtstrategien zu werten, sondern auch und gerade als ein Versuch zu verstehen, spezifische globale Einflussmöglichkeiten im „Allgemeininteresse“16 zu verbessern. Geopolitische Dienstleistungen sowie die Bemühungen zur Schaffung einer internationalen Ordnung lassen sich dabei nicht hinreichend aus mächtigen Profitinteressen erklären, sondern sind immer auch Ausdruck der Interessen einzelstaatlicher Instanzen, die damit etwa auf die Aufrechterhaltung ihrer Souveränität und damit ihrer Machtbasis zielen (Deudney 2000).

Die Entstehungsgrundlagen kapitalistischer Geopolitik unterscheiden sich alles in allem erheblich vom geopolitischen System des Feudalismus bzw. Absolutismus (Giddens 1987, S. 222-236). In letzterem waren Krieg und „politische Akkumulation“ (Landraub) zentrale Instrumente der Aneignung eines Mehrprodukts (Brenner 1986). Die Okkupation von „fremdem“ Territorium im Kapitalismus ist zwar vorstellbar, aber nicht zwingend notwendig: Sollte die Kontrolle von Räumen zur Ermöglichung und Absicherung ökonomischer Durchdringung auch ohne direkte Herrschaft möglich sein, ist dies im Regelfall ein befriedigender Zustand für die beteiligten Staaten bzw. Machteliten.

Es sei an dieser Stelle, überleitend zum nächsten Abschnitt, noch einmal darauf verwiesen, dass internationale Konkurrenzverhältnisse sich nicht allein aus externen Spannungen ableiten lassen, sondern erst in einer Kombination mit den innergesellschaftlichen Dynamiken, normativen Konstellationen und Kräfteverhältnissen (sowie weiteren transnationalen Dynamiken) angemessen zu untersuchen sind.

\section{Spielarten der Geopolitik im globalen Kapitalismus}

Allgemein lässt sich aus dem Vorangegangenen folgern, dass aus den vielgestaltigen Artikulationen von wirtschaftlichen und politischen Kooperations- und Konkurrenzverhältnissen konflikthaft strukturierte inter- und transnationale Räume hervorgehen. Diese Räume konstituieren ein Geflecht sozialer Beziehungen spezifischen Charakters, auf dem andere Handlungsrationalitäten als innerhalb der (in der Forschung häufig als Referenz-

15 Insofern kann die Rüstungskonkurrenz als eine zentrale Form des geopolitischen Wettbewerbs verstanden werden.

16 Staatsführungen zielen darauf ab, differierende Einzelinteressen zu einem legitimen außenwirtschaftlichen und -politischen Allgemeininteresse zu bündeln. Das leitet über zu dem Problem eines je konkret durch den Staat zu bestimmenden Interesses, d. h. der Art und Weise, wie der Einzelstaat auf variierende, internationale Herausforderungen reagiert. Dabei werden immer alternative, umkämpfte Umsetzungsmöglichkeiten politisch artikuliert, was die Untersuchung von innergesellschaftlichen Entwicklungen, u. a. von Verbänden, Parteien bzw. eine interne Differenzierung der Machteliten in Fraktionen, die Kompromisse mit anderen sozialen Gruppen einschließt, erforderlich macht. 
folie verwendeten) relativ stabilen westlichen Gesellschaften herrschen. Die Bedeutung der Dimension von Hierarchie und Unsicherheit (Hobson und Sharman 2005) auf der Ebene der internationalen Beziehungen untermauert die These, der zufolge inter- und transnationale Dynamiken Prozesse ohne steuerndes Zentrum bleiben.

Zugleich macht die stetige Veränderung moderner Gesellschaften es notwendig, das Verhältnis der Strukturmerkmale des globalen Kapitalismus zu den verschiedenen historischen Phasen der kapitalistischen Entwicklung in Beziehung zu setzen, d. h. zu der Art und Weise, wie sich die Strukturmerkmale zu typischen Merkmalskombinationen einer bestimmten historischen Periode und/oder Konstellation ausbilden (Bieler und Morton 2001). ${ }^{17}$ Es gilt dabei, den Gegensatz zwischen gesellschaftlich bedingter Handlungsfähigkeit und institutionalisierten Handlungszwängen zu überwinden. Eine angemessene Berücksichtigung der Handlungsebene kann dazu dienen, die relevante Rolle strategisch-politischer Projekte von Machteliten sowie die kontingenten Entwicklungen in den internationalen Beziehungen zu erfassen. Über die allgemeinen Bedingungen der Konfliktentstehung hinaus kann nun zu einer Analyse der historischen Wirklichkeit übergegangen werden.

Die historische Transformation der Außen(wirtschafts-)politik lässt sich nunmehr vor dem Hintergrund der sich verändernden Bedeutung der Politisierung der Ökonomie, unter anderem durch sich wandelnde Formen des staatlichen Interventionismus (etwa im liberalen Ordnungsstaat oder im stärker regulierenden Interventionsstaat) sowie durch das Mischungsverhältnis zwischen „harter“ und „weicher“ Geopolitik, genauer beschreiben.

Die weiche Spielart der Geopolitik beschränkt sich auf die Einflussnahme auf einem fremden Territorium, in internationalen Organisationen und auf ausländischen Märkten mit friedlichen Mitteln, die zu ökonomischen Abhängigkeiten führt und/oder politischen Druck ausübt. Im Rahmen weicher Geopolitik spielen politische oder diplomatische Maßnahmen, ökonomische Sanktionen und Anreize eine wichtige Rolle bei der Durchsetzung von Ambitionen im jeweiligen Zielgebiet bzw. in der Auseinandersetzung zwischen Staaten (Gritsch 2005). Die harte Spielart der Geopolitik zielt dagegen mit vorwiegend militärischen Mitteln auf eine politische Kontrolle externer Räume und/oder auf die Bewahrung bzw. Steigerung des internationalen Einflusses.

Weil die Intensität des Umgangs mit Konkurrenzen bzw. der Konfliktaustragung variiert, können die Formen der internationalen Auseinandersetzungen auf einem Kontinuum angeordnet werden. Dies reicht von den Auseinandersetzungen in den Arenen der internationalen politischen Institutionen, über handels- und währungspolitische Spannungen sowie bestimmten, weichen geopolitischen Maßnahmen wie Sanktionen, bis hin zur Androhung militärischer Gewalt bzw. der Zurschaustellung militärischer Kapazitäten und weiteren harten geopolitischen Handlungen. Sie können in der Gewaltanwendung kulminieren. ${ }^{18}$

17 Hegemoniale und nichthegemoniale Weltordnungsphasen und ihre, in einem weiteren Schritt einzuführenden, variierenden sozioökonomischen und geopolitischen Verlaufsformen können entlang der Entwicklungslinie vom klassischen Imperialismus (1870-1945) über die Weltordnungsphase des Supermachts-Imperialismus (1945-1989) bis zur Phase der neuen Weltunordnung ab 1989 nachgezeichnet werden (ten Brink 2008b, S. 136-217).

18 In den letzten zwei Jahrzehnten haben sich Formen der harten und weichen Geopolitik gewandelt. Einstmals taktische Elemente wurden zur Strategie fortentwickelt. Hierunter fällt etwa der 


\section{Ausblick}

Abschließend weise ich auf Grundlage der eingeführten Merkmale des globalen Dispositivs vieler Kapitalismen und staatlicher Strategien kursorisch auf einige bedeutende Konkurrenzbeziehungen hin, die geopolitische Dynamiken ausgelöst haben bzw. zukünftig auslösen können.

\section{(1) Multipolarität, Wettbewerbsstaatlichkeit und Funktionsstörungen in internationalen} Institutionen: Mit dem Zusammenbruch des Ostblocks, der ein geopolitisches Vakuum hinterließ - das an seinen südlichen Rändern in Zentralasien entstandene Machtvakuum entwickelte sich zu einer der wichtigsten Konfliktzonen heutiger Sicherheits- und Energiepolitik -, wurde die bipolar strukturierte Welt kurzzeitig durch die alleinige Vorherrschaft der Vereinigten Staaten abgelöst. Vor dem Hintergrund von veränderten sozioökonomischen Kräfteverhältnissen, instabilen Märkten, Konkurrenzverhältnissen im Bereich der Geld- und Währungsbeziehungen und aufsteigenden Mächten konnte der Wunsch nach einem amerikanischen Imperium allerdings nicht in eine erneute, nunmehr globale Hegemonie überführt werden (Mann 2003; Smith 2005). ${ }^{19}$ Der Prozess eines relativen Machtschwunds der USA offenbarte sich jüngst in der Finanz- und Wirtschaftskrise, die die amerikanische Volkswirtschaft wesentlich härter als frühere Krisen traf und zudem den Aufstieg Chinas wirtschaftlich und machtpolitisch akzentuierte.

Die Relevanz nicht immer komplementär handelnder einzelstaatlicher Apparate äußert sich in den letzten Jahren außenwirtschaftspolitisch in Form des gestärkten Selbstverständnisses des Wettbewerbsstaates - vor dem Hintergrund von Überakkumulationstendenzen und einer Zunahme der Konkurrenz zwischen den Unternehmen auf internationaler Ebene

offensive „Blitzkrieg“, der auf die Vermeidung langer Kriege setzt (z. B. die Jugoslawienintervention der Nato 1999), die defensive „Maginotdoktrin“, die darauf abstellt, die Verteidigungskräfte tendenziell unverwundbar zu machen und in der Diskussion um Raketenabwehrsysteme ihre aktuelle Entsprechung findet, sowie ein ,weicher“ Ansatz, der Wirtschaftsblockaden, andere Sanktionen und Druckmittel enthält. Ab den 1990er Jahren wurden ökonomische Sanktionen in den Bereichen Handel und Finanzen häufiger eingesetzt als jemals zuvor im 20. Jahrhundert (Grieco und Ikenberry 2003, S. 172).

19 Entgegen der These, dass der „Krieg gegen den Terror“ und besonders die Irakpolitik ab 2002 allein irrationalen Machtpolitiken neokonservativer Regierungseliten geschuldet war, weisen Autoren darauf hin, dass diese auch als ein riskanter (und schließlich gescheiterter) Versuch der amerikanischen Staatsführung verstanden werden kann, erstens militärische Überlegenheit und damit Hegemoniefähigkeit unter Beweis zu stellen, zweitens die Dominanz im Nahen Osten gegenüber anderen Großmächten und der OPEC auszubauen und drittens ,demokratische Revolutionen“ voranzutreiben bzw. westlich orientierte liberale Marktwirtschaften zu etablieren - Aspekte, die im Interesse großer Teile der amerikanischen Unternehmen gelegen hätten (Callinicos 2009, S. 223-227; vgl. Bromley 2005). Über die These einer Gegenreaktion des harten Kerns der Staatsorgane, des Gewaltmonopols - seit „dessen Organe zusehends von nichtstaatlichen Akteuren herausgefordert werden“ (Müller 2009, S. 241) - hinaus, besitzt die angestrebte Herstellung und Aufrechterhaltung einer hegemonialen politischen Führungsrolle erhebliche Vorzüge für die amerikanische Volkswirtschaft. Kirshner (2008) beschreibt dies am Beispiel der globalen Vorherrschaft des US-Dollars. Diesem Umstand verdankt der amerikanische Staat größere Kapazitäten als andere Nationen, Leistungsbilanzdefizite, einen gigantischen Verteidigungshaushalt sowie Krisen zu schultern. 
(Brenner 2006, S. 239-249). Der nationale Standortwettbewerb des 21. Jahrhunderts ist insofern keine Fehlwahrnehmung, sondern basiert auf der Existenz vieler wirtschaftlicher Zentren, die wiederum rückgebunden sind an die jeweiligen gesellschaftlichen Kräftekonstellationen sowie an die institutionell verankerten, weiterhin vorwiegend von einzelstaatlichen Herrschaftsinstanzen (wenn auch verstärkt in öffentlich-privaten Mischformen) bearbeiteten Governance-Strukturen. Hinsichtlich der makroökonomischen Einflussmöglichkeiten der Wirtschafts- und Finanzpolitik haben beispielsweise mit den Zentralbanken restrukturierte Institutionen eines Quasi-Staatsinterventionismus an Bedeutung gewonnen. Der globale Einbruch 2008/2009, der nationale Krisenlösungsstrategien zeitigte und eine Debatte um einen „neuen“ Protektionismus auslöste, den Schutz einheimischer Produzenten vor ,ausländischer“ Konkurrenz, pointierte dies noch einmal.

Auch wenn gegenwärtig ein hohes Potential zur Koordination auf supranationaler Ebene existiert, haben Tendenzen der Transnationalisierung sozialer Prozesse in der neuen Weltunordnung bislang vorwiegend zu makroregionalen Wirtschaftseinheiten (mit allenfalls partieller politischer Integration) geführt. Sie bedürfen weiterhin der staatlichinstitutionellen Regulierung und der Ressourcen der Einzelstaaten. Die Weiterentwicklung dieses Trends verbietet ökonomistische Kurzschlüsse: Auch wenn die Unternehmen transnationaler als jemals zuvor handeln und sich etwa auf die infrastrukturellen Voraussetzungen mehrerer Staaten gleichzeitig beziehen, gehen die Staatsführungen weiterhin von international wettbewerbsfähigen ,einheimischen“ Kapitalien aus und besitzen ein Interesse an dauerhaften Beziehungen zu ihnen, weil sie sich in einer strukturellen Abhängigkeit gegenüber einer gelingenden Kapitalakkumulation innerhalb ihres Territoriums befinden (ten Brink 2008b, S. 154-165).

Nationale bzw. regionale „Alleingänge“ stimulierten aus diesem Grund - und zwar bereits vor dem globalen Einbruch - Funktionsstörungen in den internationalen politischen Institutionen. Rivalitäten zwischen der EU und den USA, besonders aber zwischen dem „Norden“ und den „Schwellenländern“, bewirkten zum Beispiel seit 2003 eine schwelende Krise der Welthandelsorganisation. Zwar sind sich die politischen Machteliten der stärksten Volkswirtschaften der Erde häufig schnell einig darin, wie im Allgemeinen eine liberale globale Ordnung einzurichten sei, sie streiten aber untereinander um die konkrete Rangordnung in der Herstellung dieser Ordnung (Chorev und Babb 2009).

Während heute die aufstrebenden „BRIC“-Länder sich gewissermaßen als „Status Quo“-Mächte erst einmal in bestehende Regulierungsinstitutionen einbinden lassen und mit einer Politik der praktischen Anpassung auf eine normative wie politische Integration abzielen, deutet ein detaillierterer Blick auf einen relativ inkohärenten Regulierungszusammenhang in den internationalen Institutionen hin. Innerhalb des neuen G20-Forums selbst Ausdruck des Machtzuwachses von Schwellenländern - verhindern konfligierende Positionen und wirtschaftspolitische Strategien (etwa zur Linderung der Bankenmalaise) die Durchsetzung signifikanter globaler Re-Regulierungen, was die Wahrscheinlichkeit erneuter weltwirtschaftlicher Instabilitäten erhöht. Die Folgewirkungen des globalen Einbruchs haben ferner die EU in massive Schwierigkeiten gestürzt. Von der Zahlungsunfähigkeit bedrohte Länder der Euro-Zone lassen sogar das vor ein paar Jahren undenkbare Ende bzw. eine Verkleinerung der Währungsunion als „Lösung“ erscheinen. 
(2) Währungskonflikte: Beispielhaft für die komplexe Vermittlung von ökonomischen Konkurrenzverhältnissen und wirtschaftspolitischen Kontrollstrategien bzw. -kapazitäten stehen gegenwärtig Währungsdispute, die mittelfristig die Rolle des US-Dollars als Weltleitwährung gefährden könnten.

Nachdem, im Unterschied zu einigen Schwellenländern, der Wachstumsbilanz vieler OECD-Wirtschaften (besonders der Vereinigten Staaten) auch im Jahr 2010 Mängel attestiert werden, zielen eine Reihe entwickelter Volkswirtschaften wie Japan oder Deutschland gleichzeitig auf die Strategie des Exports ab. Um den inneren Wachstumsblockaden zu begegnen, möchte nunmehr auch die US-Administration den Export in den nächsten Jahren erheblich steigern. Sie forderte auf dem G20-Gipfel sogar eine (erfolglose) Begrenzung von Exportüberschüssen anderer Exportländer. Da in diesem Zusammenhang die Wahrscheinlichkeit einer Verschärfung der Konkurrenz um die weltweiten Exportmärkte zunimmt - unter der Voraussetzung einer Exportschwemme können Überkapazitäten entstehen; nicht alle Exportwirtschaften können daher zugleich profitieren -, versuchen einige Staaten durch eine Verbilligung der eigenen Währung Marktanteile zulasten anderer Nationen zu gewinnen. ${ }^{20}$ In der westlichen Kritik an unterbewerteten Währungen von Schwellenländern (besonders Chinas) kommt der sich verschärfende ökonomische Wettbewerb gewissermaßen politisch zum Ausdruck. ${ }^{21}$ In diesem Zusammenhang hat auch die Strategie der amerikanischen Zentralbank, eine neue Phase der „quantitativen Lockerung“ (Quantitative Easing II - QE2) zu starten - und über den Kauf von Staats- und Privatanleihen die im Umlauf befindliche Geldmenge zu steigern - vergleichsweise heftige internationale Kritik hervorgerufen. QE2 kann den Wert des Dollars senken und amerikanische Exporte verbilligen.

$\mathrm{Ob}$ sich diese Spannungen, etwa vor dem Hintergrund eines erneuten Einbruchs von Volkswirtschaften, zu schwerwiegenden politischen Auseinandersetzungen hochschaukeln, ist nicht sicher. ${ }^{22}$ Möglich wäre es, zumal, wenig beachtet, sich bereits in den Jahren vor der Krise die Hegemonialposition des US-Dollars abschwächte, was sich beispielsweise in Vorschlägen Chinas zur Schaffung einer neuen Weltfinanzarchitektur manifes-

20 Wer die Vergabe von Staatsgeldern an marode Unternehmen zugleich an Entlassungen und Lohnsenkungen knüpft, droht einen „Neomerkantilismus“ zu stimulieren, der unter Bedingungen weltweit sinkender Nachfrage in eine verschärfte Beggar-thy-neighbour-Politik umschlägt. Selbst das Gespenst der Deflation kann dann durchaus real werden.

21 Die Führung der marktliberal-staatskapitalistisch transformierten Volksrepublik China (vgl. ten Brink 2010) kontert gegen die Forderung, den Yuan aufzuwerten, mit dem Argument, dies schwäche die chinesische Wettbewerbsfähigkeit, was wiederum zu sozialen Unruhen mit negativen Folgen für ausländische Investoren führe. In diesem Zusammenhang werden nationalistische und antiwestliche Bewegungen in China angeführt, deren Radikalisierung sich in einer aggressiven Außenpolitik manifestieren könnte (vgl. Shirk 2008). Es gilt aus diesem Grund zu untersuchen, ob sich die chinesische Staatsführung nach außen zielender Bedrohungskonstruktionen bedient.

22 Sollte die Volksrepublik ihre Dollarbestände schnell umschichten, würde deren Wert fallen und zugleich der Wert des chinesischen Yuan steigen, was einen zentralen Wettbewerbsvorteil der Exportökonomie Chinas konterkariert. Die Staatsführung denkt mit ihren Vorschlägen daher vorwiegend an einen mittel- bis längerfristigen Prozess der Umschichtung, der die Erosion der aktuellen Leitwährung vorantreiben würde. 
tierte (Herr und Hübner 2005; McNamara 2008; Cohen 2009). Die in den letzten Jahren mit dem Begriff „Bretton Woods II“ umschriebene Konstellation, die auf Leistungsbilanzdefiziten der USA einerseits und Leitungsbilanzüberschüssen in Ostasien andererseits basierte und die Dominanz des US-Dollars trotz der hohen Staats- und Auslandsverschuldung der Vereinigten Staaten garantierte, wird angezweifelt, und ein fragmentiertes Währungssystem wahrscheinlicher. Da dies nicht im Interesse der USA liegt, deutet diese Entwicklung auf zukünftige Konflikte hin.

Begleitet von diplomatischen Beschwichtigungsgesten führt die Obama-Administration gegenwärtig, anknüpfend an liberal-realistische Traditionslinien, Sicherheitsmaßnahmen fort, um China in eine Art Konzert der Mächte einzuordnen - dirigiert durch die USA, mit dem Ziel einer Unterordnung bzw. Domestizierung der Volksrepublik (Kaplan 2010). Dass die gegenwärtig nur episodisch stattfindenden Auseinandersetzungen mit China von der aktuellen (oder zukünftigen) US-Administration - wohlwissend um die wechselseitigen Abhängigkeiten zwischen den zwei größten Volkswirtschaften der Erde - in manifeste geopolitische Konflikte ausgeweitet werden, erscheint dennoch nicht völlig abwegig. Sollten sich die Anhaltspunkte für eine Erosion des amerikanisch dominierten Regulierungsgeflechts auf globaler Ebene verdichten und Konflikte jenseits der Wirtschafts- und Geldpolitik zunehmen, beispielsweise in Fragen der Ressourcensicherung, erscheint dies möglich.

Zusätzlich könnten weitere innergesellschaftliche Triebfedern die Rivalitäten befördern, etwa Versuche einer Krisenexternalisierung. Bereits die Radikalisierung der Außenpolitik der Vereinigten Staaten unter Bush stellte zu einem Teil den Versuch dar, soziale Widersprüche des amerikanischen Wachstumsmodells zu überlagern (Glassman 2005). Vor dem Hintergrund des Scheiterns der neokonservativen Strategien zielt die ObamaAdministration nunmehr auf eine Wiederherstellung und Erneuerung der amerikanischen Führungsrolle in der Welt ab (De Graaff und van Apeldoorn 2011). Welche strategische Ausrichtung sich im Verhältnis zu China etabliert, hängt dabei nicht zuletzt vom Ausgang inneramerikanischer Auseinandersetzungen ab: Während tief in die chinesische Wirtschaft eingebettete transnationale US-Konzerne an kooperativen Beziehungen mit China interessiert sind, stehen dem Kapitalgruppen skeptisch gegenüber, die sich stärker am amerikanischen Binnenmarkt orientieren. Zudem existieren politische Auseinandersetzungen zwischen populistisch-protektionistischen Kräften (u. a. in der „Tea-Party-Bewegung") und einem vorwiegend transnational ausgerichteten Machtblock. Sollten sich erstere Kräfte durchsetzen, droht eine konfrontativere Haltung der US-Regierung - eine Möglichkeit, die gerade aufgrund der Instabilitäten der amerikanischen Wirtschaft nicht unwahrscheinlich ist: „The United States now faces the prospect of a prolonged period of economic difficulties, which increases the possibility of looking upon China as the scapegoat. Ever since independence, the United States has had a long history of achieving internal political stability by uniting the nation against a perceived external threat" (Nolan 2010, S. 86; vgl. ten Brink und Lindemann 2012).

Zweifellos darf die Konflikthaftigkeit der aktuellen Weltordnungsphase nicht als eine einfache Wiederholung der historischen Phase nach 1929 interpretiert werden. Die gegenwärtig unwahrscheinliche Eskalation von Rivalitäten zwischen den stärksten Staaten (insbesondere innerhalb des NATO-Bündnisses) in manifeste zwischenstaatliche Militärkonflikte darf jedoch nicht zur Vernachlässigung der vielschichtigen Konkurrenz- und Konfliktformen unterhalb der Schwelle des zwischenstaatlichen Kriegs führen. 
Auch wenn verschiedene Gesellschaften in wirtschaftspolitischer Hinsicht ein im Kern ähnliches marktliberales policy regime durchsetzen, bedeutet das im Umkehrschluss nicht, dass unter ihnen keine ernsthaften Interessengegensätze bestehen oder entstehen können. Wenn der Unternehmenswettbewerb in eine politisch vermittelte Standortkonkurrenz und Währungskonflikte mündet, politisches Handeln im Rahmen einer fragmentierten, krisengeschüttelten Weltwirtschaft noch unsicherer als zuvor erscheint und sich divergierende Krisenlösungsstrategien gegenüberstehen, erzeugen diese Konstellationen Spielräume für destruktive Konkurrenzbeziehungen, nationalistische Bewegungen und zwischenstaatliche Rivalitäten, die geopolitisches Konfliktpotential in sich tragen. Auf bedenkliche Art und Weise konterkariert das den erwarteten gesellschaftlichen Selbstaufklärungs- bzw. Rationalisierungsprozess in der Moderne und verringert die Aussichten auf eine harmonische Global Governance oder ein Konzert der Mächte.

\section{Literatur}

Anderson, B. (1996). Die Erfindung der Nation. Zur Karriere eines folgenreichen Konzepts. Frankfurt: Campus.

Beck, U. (2004). Der kosmopolitische Blick oder: Krieg ist Frieden. Frankfurt: Suhrkamp.

Bieler, A., \& Morton, A. D. (2001). The gordian knot of agency-structure in international relations: A neo-gramscian perspective. European Journal of International Relations, 7(1), 5-35.

Block, F. (1994). The roles of the state in the economy. In N. Smelser \& R. Swedberg (Hrsg.), Handbook of economic sociology (S. 691-710). Princeton: Princeton University Press.

Block, F. (2005). Towards a new understanding of economic modernity. In C. Joerges, B. Strath, \& P. Wagner (Hrsg.), The economy as a polity. The political constitution of contemporary capitalism (S. 3-16). London: UCL Press.

Boyer, R. (2005). How and why capitalisms differ. Economy and Society, 34(4), 509-557.

Brand, U. (2006). Die politische Form der Globalisierung. Politische Institutionen und soziale Kräfte im internationalisierten Staat. Habilitationsschrift, Universität Kassel.

Brenner, R. (1986). The social basis of economic development. In J. Roemer (Hrsg.), Analytical marxism (S. 23-53). Cambridge: Cambridge University Press.

Brenner, R. (2006). The economics of global turbulence. The advanced capitalist economies from long boom to long downturn, 1945-2005. London: Verso.

Brock, L. (2006). Kriege der Demokratien. Eine Variante des Demokratischen Friedens. In A. Geis (Hrsg.), Den Krieg überdenken. Kriegsbegriffe und Kriegstheorien in der Kontroverse (S. 203-228). Baden-Baden: Nomos.

Bromley, S. (2005). The united states and the control of world oil. Government and Opposition, $40(2), 225-255$.

Callinicos, A. (2007). Does capitalism need the state system? Cambridge Review of International Affairs, 20(4), 533-549.

Callinicos, A. (2009). Imperialism and global political economy. Cambridge: Polity Press.

Chibber, V. (2004). The return of imperialism to social science. Archives de Européennes de Sociologie - The European Journal of Sociology, 45(3), 427-441.

Chorev, N., \& Babb, S. (2009). The crisis of neoliberalism and the future of international institutions: A comparison of the IMF and the WTO. Theory and Society, 38(5), 459-484.

Cohen, B. J. (2000). The geography of money. Ithaka: Cornell University Press.

Cohen, B. J. (2009). The future of reserve currencies. Finance \& Development, 9, 26-29.

Cox, R. W. (1987). Production, power, and world order. Social forces in the making of history. New York: Columbia University Press.

Cox, R. W., \& Schechter, M. G. (2002). The political economy of a plural world. London: Routledge. 
De Graaff, N., \& van Apeldoorn, B. (2011). Varieties of US post-cold war imperialism: Anatomy of a failed hegemonic project and the future of US geopolitics. Critical Sociology, 37(4), 403-427.

Deudney, D. (2000). Geopolitics as theory: Historical security materialism. European Journal of International Relations, 6(1), 77-107.

Deutschmann, C. (2009). Soziologie kapitalistischer Dynamik (Max-Planck-Institut Scholar in Residence Lectures). http://www.mpifg.de/aktuelles/SiR/MPIfG-Deutschmann_0902.pdf. Zugegriffen: 25. Juli 2009.

Geis, A. (2008). Andere, Fremde, Feinde. Bedrohungskonstruktionen in der Demokratie. In A. Brodocz, M. Llanque, \& G. S. Schaal (Hrsg.), Bedrohungen der Demokratie (S. 170-188). Wiesbaden: VS Verlag für Sozialwissenschaften.

Giddens, A. (1987). The nation-state and violence. Volume two of a contemporary critique of historical materialism. Berkeley: University of California Press.

Glassman, J. (2005). The new imperialism? On continuity and change in US foreign policy. Environment and Planning A, 37(9), 1527-1544.

Görg, C. (2002). Einheit und Verselbständigung. Probleme einer Soziologie der „Weltgesellschaft“. Zeitschrift für Internationale Beziehungen, 9(2), 275-304.

Grieco, J. M., \& Ikenberry, J. G. (2003). State power and world markets. The international political economy. New York: W.W. Norton.

Gritsch, M. (2005). The nation-state and economic globalization: Soft geo-politics and increased state autonomy? Review of International Political Economy, 12(1), 1-25.

Hardt, M., \& Negri, A. (2002). Empire - Die neue Weltordnung. Frankfurt: Campus.

Harvey, D. (2005). Der neue Imperialismus. Hamburg: VSA.

Harvey, D. (2010). The enigma of capital: And the crises of capitalism. London: Profile Books.

Hay, C. (2005). Globalisation's impact on states. In J. Ravenhill (Hrsg.), Global political economy (S. 235-262). Oxford: Oxford University Press.

Held, D., \& McGrew, A. (Hrsg.). (2002). Governing globalization. Power, authority and global governance. Cambridge: Polity Press.

Herr, H., \& Hübner, K. (2005). Währung und Unsicherheit in der globalen Ökonomie. Eine geldwirtschaftliche Theorie der Globalisierung. Berlin: Edition Sigma.

Hirsch, J. (2005). Materialistische Staatstheorie. Transformationsprozesse des kapitalistischen Staatensystems. Hamburg: VSA.

Hobson, J. M., \& Sharman, J. C. (2005). The enduring place of hierarchy in world politics: Tracing the social logics of hierarchy and political change. European Journal of International Relations, 11(1), 63-98.

Jessop, B. (2004). Spatial fixes, temporal fixes and spatio-temporal fixes. http://www.lancs.ac.uk/ fass/sociology/papers/jessop-spatio-temporal-fixes.pdf. Zugegriffen: 20. Juli 2007.

Jessop, B. (2007). State power. A strategic-relational approach. Cambridge: Polity Press.

Kaplan, R. D. (2010). The geography of chinese power. How far can Beijing reach on land and at sea? http://www.foreignaffairs.com/articles/66205/robert-d-kaplan/the-geography-of-chinesepower. Zugegriffen: 20. Aug. 2010.

Kirshner, J. (2008). Dollar primacy and American power: What's at stake? Review of International Political Economy, 15(3), 418-438.

Lacher, H. (2005). International transformation and the persistence of territoriality: Toward a new political geography of capitalism. Review of International Political Economy, 12(1), 26-52.

Mann, M. (1990). Geschichte der Macht. Erster Band. Von den Anfängen bis zur griechischen Antike. Frankfurt: Campus.

Mann, M. (1997). Hat die Globalisierung den Siegeszug des Nationalstaates beendet? Prokla, 106, $113-141$.

Mann, M. (2003). Die ohnmächtige Supermacht. Warum die USA nicht die Welt regieren können. Frankfurt: Campus.

McNamara, K. R. (2008). A rivalry in the making? The euro and international monetary power. Review of International Political Economy, 15(3), 439-459. 
Mearsheimer, J. J. (2003). The tragedy of great power politics. New York: W.W. Norton.

Möller, K. (2010). Die Logik einer kapitalistischen Welt? Die Transformation der Sicherheitsarchitektur in der Perspektive der kritischen internationalen politischen Ökonomie. In A. FischerLescano \& P. Mayer (Hrsg.), Die Transformation von Recht und Politik globaler Sicherheit. Entwicklungstendenzen und Interpretationen. Frankfurt: Campus.

Mosley, L. (2003). Global capital and national governments. Cambridge: Cambridge University Press.

Mouffe, C. (2007). Über das Politische. Wider die kosmopolitische Illusion. Frankfurt: Suhrkamp.

Müller, H. (2009). Staatlichkeit ohne Staat - ein Irrtum aus der europäischen Provinz? Limitierende Bedingungen von Global Governance in einer fragmentierten Welt. In N. Deitelhoff \& J. Steffek (Hrsg.), Was bleibt vom Staat? Demokratie, Recht und Verfassung im globalen Zeitalter (S. 221-258). Frankfurt: Campus.

Nolan, P. (2010). America and the crossroads of capitalist globalization. Challenge, 53(6), 77-92.

Offe, C., \& Ronge, V. (1976). Thesen zur Begründung des Konzepts des „kapitalistischen Staates“ und zur materialistischen Politikforschung. In C. Pozzoli (Hrsg.), Rahmenbedingungen und Schranken staatlichen Handelns (S. 54-70). Frankfurt: Suhrkamp.

Ohmae, K. (1995). The end of the nation state. New York: The Free Press.

Gindin, S., \& Panitch, L. (2004). Globaler Kapitalismus und amerikanisches Imperium. Hamburg: VSA.

Rosenberg, J. (2005). Globalization theory: A post mortem. International Politics, 42(1), 2-74.

Rosenberg, J. (2006). Why is there no international historical sociology? European Journal of International Relations, 12(3), 307-340.

Shirk, S. L. (2008). China. Fragile superpower. How china's internal politics could derail its peaceful rise. Oxford: Oxford University Press.

Smith, N. (2005). The endgame of globalization. London: Routledge.

Strange, S. (1996). The retreat of the state: The diffusion of power in the world economy. Cambridge: Cambridge University Press.

Streeck, W. (2009). Institutions in history. Bringing capitalism back in. http://www.mpifg.de/pu/ mpifg_dp/dp09-8.pdf. Zugegriffen: 30. Jan. 2010.

ten Brink, T. (2008a). Staatenkonkurrenz. Zur Analyse von Geopolitik und Imperialismus - ein Überblick. Stuttgart: UTB.

ten Brink, T. (2008b). Geopolitik. Geschichte und Gegenwart kapitalistischer Staatenkonkurrenz. Münster: Westfälisches Dampfboot.

ten Brink, T. (2010). Strukturmerkmale des chinesischen Kapitalismus. http://www.mpifg.de/pu/ mpifg dp/dp10-1.pdf. Zugegriffen: 20. Feb. 2010.

ten Brink, T. (Hrsg.) (2011). Globale Rivalitäten. Staat und Staatensystem im globalen Kapitalismus. Stuttgart: Franz Steiner Verlag.

ten Brink, T., \& Lindemann, T. (2012). Globalization, misrecognition, and „terrorist“ violence. In H. Tigroudja \& L. Hennnebel (Hrsg.), Balancing Liberty and Security: The Human Rights Pendulum (S. 241-256). Den Haag: Wolf Legal Publishers.

Teschke, B. (2003). The myth of 1648. Class, geopolitics and the making of modern international relations. London: Verso.

Teschke, B., \& Lacher, H. (2007). The changing ,logics' of capitalist competition. Cambridge Review of International Affairs, 20(4), 565-580.

van der Pijl, K. (2006). Global rivalries from the cold war to Iraq. London: Pluto Press.

van der Pijl, K. (2007). Capital and the state system: A class act. Cambridge review of international affairs, 20(4), 619-637.

Wendt, A. (2003). Why a world state is inevitable. European journal of international relations, 9(4), 491-542.

Wolf, R. (2004). Macht und Recht in der ZIB. Zeitschrift für Internationale Beziehungen, 11(2), $313-320$.

Wood, E. M. (2003). Empire of capital. London: Verso. 